\title{
Tumor and Endothelial Cell Hybrids Participate in Glioblastoma Vasculature
}

\author{
Soufiane El Hallani, ${ }^{1}$ Carole Colin, ${ }^{2}$ Younas El Houfi, ${ }^{1}$ Ahmed Idbaih, ${ }^{1,3}$ \\ Blandine Boisselier, ${ }^{1}$ Yannick Marie, ${ }^{1}$ Philippe Ravassard, ${ }^{1}$ Marianne Labussière, ${ }^{1}$ \\ Karima Mokhtari, ${ }^{4}$ Jean-Léon Thomas, ${ }^{1}$ Jean-Yves Delattre, ${ }^{1,3}$ \\ Anne Eichmann, ${ }^{5}$ and Marc Sanson ${ }^{1,3}$ \\ ${ }^{1}$ Sorbonne Universités, UPMC, Université Paris 06, Inserm, CNRS, UM 75, U 1127, UMR 7225, ICM, 75013 Paris, France \\ ${ }^{2}$ UMR911-CRO2, Faculté de Médecine de la Timone, Université de la Méditerranée, 13000 Marseille, France \\ ${ }^{3}$ AP-HP, Groupe Hospitalier Pitié-Salpêtrière, Service de Neurologie Mazarin, 75013 Paris, France \\ ${ }^{4}$ AP-HP, Groupe Hospitalier Pitié-Salpêtrière, Service de Neuropathologie Raymond Escourolle, 75013 Paris, France \\ ${ }^{5}$ INSERM U833, Collège de France, 75005 Paris, France
}

Correspondence should be addressed to Marc Sanson; marc.sanson@psl.aphp.fr

Received 11 February 2014; Accepted 2 March 2014; Published 24 April 2014

Academic Editor: Giuseppe Lombardi

Copyright (C) 2014 Soufiane El Hallani et al. This is an open access article distributed under the Creative Commons Attribution License, which permits unrestricted use, distribution, and reproduction in any medium, provided the original work is properly cited.

\begin{abstract}
Background. Recently antiangiogenic therapy with bevacizumab has shown a high but transient efficacy in glioblastoma (GBM). Indeed, GBM is one of the most angiogenic human tumors and endothelial proliferation is a hallmark of the disease. We therefore hypothesized that tumor cells may participate in endothelial proliferation of GBM. Materials and Methods. We used EGFR FISH Probe to detect EGFR amplification and anti-CD31, CD105, VE-cadherin, and vWF to identify endothelial cells. Endothelial and GBM cells were grown separately, labeled with GFP and DsRed lentiviruses, and then cocultured with or without contact. Results. In a subset of GBM tissues, we found that several tumor endothelial cells carry EGFR amplification, characteristic of GBM tumor cells. This observation was reproduced in vitro: when tumor stem cells derived from GBM were grown in the presence of human endothelial cells, a fraction of them acquired endothelial markers (CD31, CD105, VE-cadherin, and vWF). By transduction with GFP and DsRed expressing lentiviral vectors, we demonstrate that this phenomenon is due to cell fusion and not transdifferentiation. Conclusion. A fraction of GBM stem cells thus has the capacity to fuse with endothelial cells and the resulting hybrids may participate in tumor microvascular proliferation and in treatment resistance.
\end{abstract}

\section{Introduction}

Glioblastomas (GBM) are the most frequent and malignant primary brain tumors in adults with poor prognosis despite surgery and conventional radio-chemotherapy. Histologically, GBM are highly angiogenic and characterized by microvascular proliferations (previously called endothelial cell proliferations) typically consisting on multilayered tufts of mitotically active endothelial cells together with smooth muscle cells and pericytes [1]. Among targeted therapies tested to date, only antiangiogenic drugs and particularly anti-VEGF have shown efficacy with a nearly $50 \%$ of responders $[2,3]$. However, this effect is always transient suggesting that GBM can acquire secondary antiangiogenic resistance. Therefore, understanding tumor endothelial cell abnormalities is important to optimize therapy. It is well established that tumor blood vessels differ from normal vessels by altered morphology, blood flow, permeability, and basement membrane deposition [4-7]. Furthermore, evidence indicates that tumor endothelial cells overexpress specific genes, proliferate rapidly, and are sensitive to growth factors and resistant to chemotherapeutic drugs [8-12]. Surprisingly, tumor endothelial cells can harbor the same chromosomal abnormality as tumor cells in B-cell lymphomas [13], multiple myeloma [14], and neuroblastoma [15], suggesting a tumor origin of at least a fraction of intratumoral endothelial cells. 
Another subpopulation of tumor cells possesses characteristics associated with normal stem cells, specifically the ability to give rise to all cell types found in a particular cancer sample [16]. In brain tumors, including GBM, tumor stem cells have been shown to possess marked capacity for proliferation, self-renewal, and differentiation into all neural lineages [17]. It has also been suggested that normal mouse neural stem cells cocultured with human endothelial cells convert into endothelial cells by transdifferentiation [18].

We here investigated whether endothelial cells of tumor origin might be present in human GBM samples and whether these cells derive from GBM stem cells. Analysis of EGFR amplified human GBM tissues by fluorescent in situ hybridization (FISH) combined with immunophenotyping [15] showed rare endothelial cells exhibiting EGFR amplification. We then investigated the capacity of GBM stem cells (GSC) to acquire an endothelial phenotype in vitro and demonstrate that this property results from cell fusion and not transdifferentiation.

\section{Materials and Methods}

2.1. GBM Tissue Preparation. Formalin-fixed, paraffin-embedded tissue sections $(5 \mu \mathrm{m})$ from 10 GBM tumors (World Health Organization classification of brain tumors [1]) carrying EGFR amplification identified by CGHa analysis [19] were deparaffinized twice with xylene. The slides were subsequently rehydrated in a series of ethanol solution $(100 \%, 90 \%$, and $70 \%$ ), washed with phosphate-buffered saline (PBS), and treated with antigen retrieval solution (citrate buffer $\mathrm{pH} 9.0$; Dako Cytomation, France) at $96^{\circ} \mathrm{C}$ for 20 minutes.

\subsection{Fluorescent In Situ Hybridization and Immunofluores-} cence. Briefly, slides were blocked with $\mathrm{PBS} / 3 \%$ bovine serum albumin (BSA) for 20 minutes and immunostained with mouse anti-human CD31 (1:20; Dako Cytomation, France) for 30 minutes at room temperature. Alexa 488conjugated goat anti-mouse antibody (1:1000; Molecular Probes, Invitrogen, France) was added as secondary reagent. After immunostaining, slides were washed three times for 5 minutes each in PBS containing 0.5\% Tween-20 (SigmaAldrich, France). Only sections of high morphologic quality were used for fluorescent in situ hybridization (FISH). The EGFR FISH Probe Mix (Dako Cytomation, France) was used according to the manufacturer's instructions. Slides were dehydrated through a series of ethanol washes $(70 \%$, $90 \%$, and $100 \%$ ), denatured in the presence of the specific probes at $82^{\circ} \mathrm{C}$ for 5 minutes, and incubated overnight in a humid chamber at $45^{\circ} \mathrm{C}$. Posthybridization washes were performed, and the slides were mounted in antifade medium Fluoromount-G (Interchim, France) with DAPI (Sigma-Aldrich, France). Slides were analyzed using a Zeiss AxioImager.Z1 microscope.

\subsection{Cell Cultures}

2.3.1. Culture of Primary GBM Stem Cells and Sphere Forming Assay. GBM samples were provided by the local Department of Neurosurgery from informed and consenting patient, as approved by the local Research Ethics Boards at the Salpetriere Hospital. Histologic analyses were done by the Department of Neuropathology. Samples were washed with Hanks' balanced salt solution (Invitrogen, France), dissected, sectioned, and enzymatically dissociated with both $5 \mathrm{mg} / \mathrm{mL}$ of Trypsin (Sigma-Aldrich, France) and $200 \mathrm{U} / \mathrm{mL}$ of DNAse (Sigma-Aldrich, France) for 10 minutes at $37^{\circ} \mathrm{C}$. Erythrocytes were lysed using $\mathrm{NH}_{4} \mathrm{Cl}$. Cells were then seeded into T75 flasks at 10000 cells $/ \mathrm{cm}^{2}$. Culture medium (neurosphere medium) consisted of DMEM/F12 (Invitrogen, France) supplemented with $20 \mathrm{ng} / \mathrm{mL}$ of epidermal growth factor (EGF), $20 \mathrm{ng} / \mathrm{mL}$ of basic fibroblast growth factor (bFGF; both from Sigma-Aldrich, France), B27 (1:50; Invitrogen, France), and $1 \%$ Penicillin/Streptomycin. Cultures were incubated in 5\% $\mathrm{CO}_{2}$ at $37^{\circ} \mathrm{C}$. After 3 days of culture, CD133 Microbead Kit (Miltenyi Biotech, France) was used to isolate $\mathrm{CD}_{133^{+}}$tumor cell population according to the manufacturer's instructions. Sorted cells were resuspended in neurosphere medium and maintained in $5 \% \mathrm{CO}_{2} / 95 \% \mathrm{O}_{2}$ atmosphere at $37^{\circ} \mathrm{C}$. Formed primary spheres were harvested, dissociated into single cells, and plated at the density of 5000 cells $/ \mathrm{cm}^{2}$ in neurosphere medium. Cultures were fed by changing half of the medium every 3 days. Subsphere-forming assay (also called passage) was repeated every 10 days.

2.3.2. Human Endothelial Cell Culture. Purified human umbilical vein endothelial cells (HUVEC) and human umbilical artery endothelial cells (HUAEC) were obtained from Promocell. Immortalized human cerebral microvascular endothelial cells (hCMEC) were obtained from Dr. Pierre Olivier Couraud (Institut Cochin, France). Endothelial cells were cultured with endothelial cell growth medium EGM-2 (Lonza, France) that contains vascular endothelial growth factor (VEGF), basic fibroblast growth factor (bFGF), epidermal growth factor (EGF), insulin growth factor I (IGFI), hydrocortisone, ascorbic acid, and $2 \%$ fetal bovine serum.

2.4. Differentiation Assay of Tumor Spheres. Primary GBM spheres were plated onto sterile multiwell glass slide coated with poly-L-ornithine (Sigma-Aldrich, France) in neurosphere medium lacking EGF and bFGF but supplemented with $10 \%$ fetal bovine serum. Cells were fixed after 7 days of differentiation culture with $4 \%$ paraformaldehyde for 15 minutes, blocked with PBS/3\% BSA for 20 minutes, and immunostained for 1 hour with primary antibodies against nestin (1:200; mouse monoclonal IgG1; Santa Cruz Biotechnology, Germany), GFAP (1:400; rabbit polyclonal; Dako Cytomation, France), and neuronal class III $\beta$-tubulin (Tuj1; 1:500; mouse monoclonal IgG2a; Covance, France). After washes, appropriate secondary antibodies were incubated for 1 hour (1:1000; Alexa 594 goat anti-mouse IgG1, Alexa 488 goat anti-rabbit and Alexa 594 goat anti-mouse IgG2a from Molecular Probes, Invitrogen, France). DAPI (SigmaAldrich, France) was used for nuclei staining. Slides were mounted in antifade medium Fluoromount-G (Interchim, France) and examined under a Zeiss AxioImager.Z1 microscope. 


\subsection{Endothelial and GBM Cells Cocultures}

2.5.1. Noncontact Coculture. Cocultures were prepared on Transwell inserts with $0.4 \mu \mathrm{m}$ pore size and $6.5 \mathrm{~mm}$ diameter (Corning Incorporated, France) placed in 24 well plates. Briefly, GFP-GSC ( $\mathrm{p} 3$ ) were seeded at $2 \times 10^{4}$ cells per well in the bottom wells of Transwells, and human endothelial cells were seeded at a ratio of 1:1 in the membrane insert wells. Cells were cultured with EGM-2 medium for 5 days. Cell layer fractions were analyzed using Nikon Eclipse TE2000U fluorescence inverted microscope.

2.5.2. Direct Contact Coculture. Dissociated human endothelial cells $\left(5 \times 10^{5}\right)$ and GFP-GSC $\left(5 \times 10^{5}\right)$ were mixed in EGM2 medium and seeded into T75 flask. After 3 days of culture, cells were dissociated, plated onto sterile multiwell glass slides $\left(1 \times 10^{4}\right.$ on an 8 -well slide $)$, and allowed to adhere for 24 hours prior to staining.

2.6. Endothelial Markers Immunofluorescence. After 3-5 days, coculture medium was washed away for 10 minutes with PBS before fixation with $4 \%$ paraformaldehyde for 15 minutes. Fixed cells were blocked with PBS/3\% BSA for 20 minutes and permeabilized for intracytoplasmic antigen with $\mathrm{PBS} / 0.1 \%$ Triton X-100. The following primary antibodies were incubated in blocking solution at room temperature for 1 hour: CD31 (1:50; mouse monoclonal IgG1; Dako Cytomation, France), CD105 (1:5; mouse monoclonal IgG1; Dako Cytomation, France), VE-cadherin (1:50; mouse monoclonal IgG1; eBioscience, France), and vWF (1:50; mouse monoclonal IgG1; Dako Cytomation, France). Following 3 washes, Alexa 594 goat anti-mouse IgG (1:1000; Molecular Probes, Invitrogen, France) was incubated in blocking solution at room temperature for 1 hour as secondary antibody. Nuclei were stained with DAPI (Sigma-Aldrich, France). Slides were mounted in antifade medium Fluoromount-G (Interchim, France) and examined under a Zeiss AxioImager.Z1 microscope. Fluorescence images were captured using AxioCam MRm camera and analyzed with AxioVision Rel. 4.6 software (Carl Zeiss).

2.7. Lentiviral Infection. GFP and DsRed lentivirus vector construction and virus production were performed by Dr. Philippe Ravassard (Pierre and Marie Curie University) as previously described [20]. Dissociated GSC were infected with GFP-expressing retroviral vector and HUVEC were infected with DsRed-expressing retroviral vector. Labeled cells were selected using a fluorescence-activated cell sorter (FACS Aria, BD Biosciences). The efficiency of transduction was over $80 \%$.

2.8. Cell Sorting. Freshly dissociated cells from GSC-GFP and HUVEC-DsRed cocultures were resuspended in $\mathrm{PBS} / 0.5 \% \mathrm{BSA} / 2 \mathrm{mM}$ EDTA solution. Labeled cells were sorted using a fluorescence-activated cell sorter (FACS Aria, BD Biosciences) on the basis of single-cell viability and the presence of double-positive GFP and DsRed fluorescence.
2.9. Reverse Transcription-PCR. Total RNA was extracted from GSC and hCMEC using RNable (Eurobio, France) and verified by electrophoresis on Agilent 2100 Bioanalyzer (Agilent Technologies, France). cDNA was synthesized with 200 units of M-MLV Reverse Transcriptase (Invitrogen, France) in $15 \mu \mathrm{L}$ of $1 \mathrm{x}$ first strand buffer (Promega, France), $2 \mathrm{mmol} / \mathrm{L}$ deoxynucleotide triphosphates in the presence of 40 units RNase inhibitor RNasin (Promega), $0.5 \mu \mathrm{g}$ random primers (Promega), and $1 \mu \mathrm{g}$ total RNA. Semiquantitative PCR amplifications were done with the following primer sequences: CD31 forward $5^{\prime}$-TCCGGATCTATGACTCAGGG-3' and reverse $5^{\prime}$-ACAGTTGACCCTCACGATCC- $3^{\prime}$; VE-cadherin forward $5^{\prime}$-TCCTCTGCATCCTCACTATCACA- $3^{\prime}$ and reverse $5^{\prime}$-GTAAGTGACCAACTGCTCGTGAA- $3^{\prime}$; ALAS forward $5^{\prime}$-TGCAGTCCTCAGGGCAGTCT$3^{\prime}$ and reverse $5^{\prime}$-TGGCCCCAACTTCCATCAT- $3^{\prime}$ as control. The PCR conditions were as follows: 5 minutes at $94^{\circ} \mathrm{C}$ for denaturation, followed by 30 seconds at $94^{\circ} \mathrm{C}, 1 \mathrm{~min}$ at $60^{\circ} \mathrm{C}$, and $1 \mathrm{~min} 30 \mathrm{sec}$ at $72^{\circ} \mathrm{C}$ for 35 cycles and $7 \mathrm{~min}$ at $72^{\circ} \mathrm{C}$ for final elongation. The RT-PCR products were electrophoretically analyzed in $1 \%$ agarose and visualized by ethidium bromide staining.

\section{Results}

3.1. Several Intratumoral Endothelial Cells Harbor EGFR Amplification. We analyzed paraffin sections from $10 \mathrm{GBM}$ with EGFR amplification, retrieved from our CGHa database [19], using CD31 monoclonal antibody to identify endothelial cells and FISH detection of EGFR amplification to identify tumor cells. As shown in Figure 1(a), EGFR amplification was detected as double minutes by red hybridization signals in a large proportion of cells present in GBM tissues. CD31 monoclonal antibody stained endothelial cells but not tumor cells (Figure 1(b)). Only 6 out of 10 GBM sections presented dense microvascular network with high morphologic quality after CD31 immunofluorescence and were then subjected to EGFR FISH. Endothelial cells carrying EGFR amplification, as shown in Figures 1(c) and 1(d), were identified in 3 out of these 6 GBM tumors. This event was episodic as we were able to count less than a hundred of CD31-EGFR FISH costained cells per section. Thus, we identified a minority of tumor endothelial cells that could derive from primary GBM cells.

3.2. Glioblastoma Stem Cells Acquire an Endothelial Phenotype When Cocultured with Human Endothelial Cells. We established 3 GBM stem cell primary cultures (GSC-1, GSC2 , and GSC-3) that demonstrated growth into tumor spheres (Figure 2(a)). They were generated from solid primary adult GBMs carrying EGFR amplification and showed conservative genomic profile in culture (Figure 2(b)). Undifferentiated tumor spheres immunostained for nestin (a characteristic neural stem cell marker) and revealed multilineage potential (expression of GFAP for astrocytes and TUJ-1 for neurons) in the differentiation assay as shown in Figure 2(d). We next explored the ability of GSC to transdifferentiate into endothelial cells. None of them expressed or stained with CD31 and 


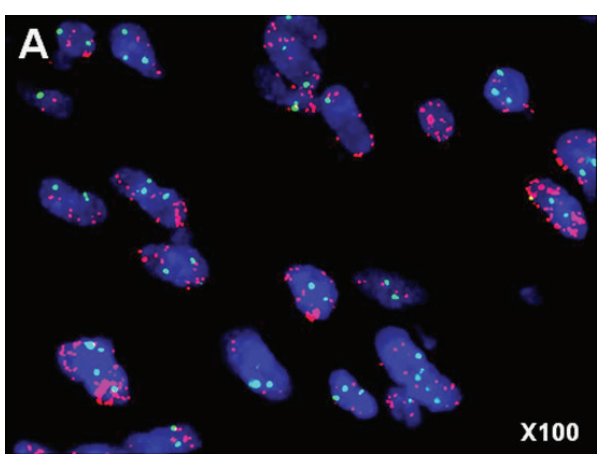

(a)

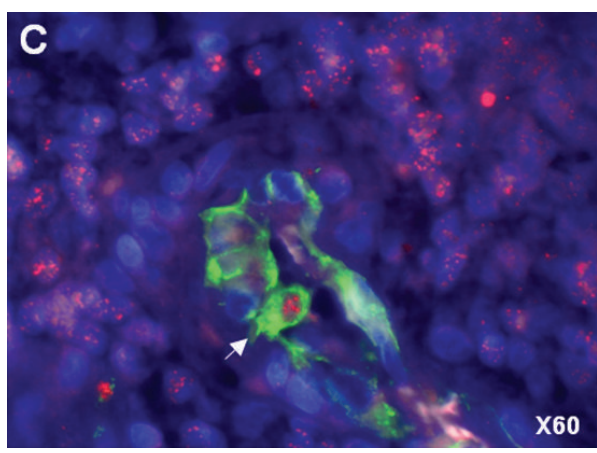

(c)

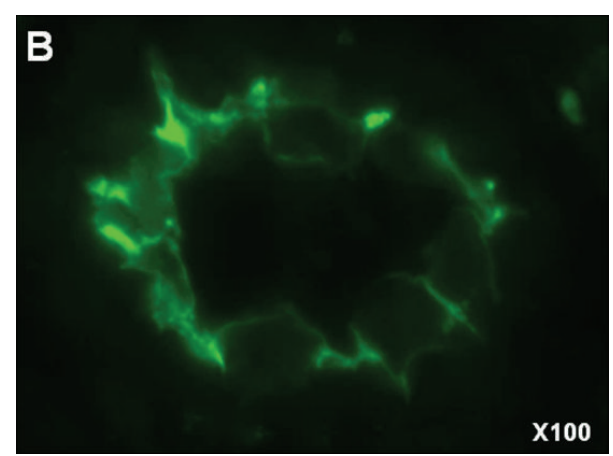

(b)

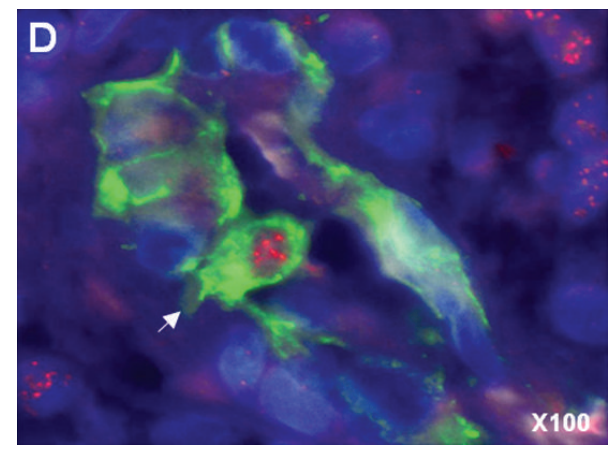

(d)

FIGURE 1: Glioblastoma-derived endothelial cells. (a) Nuclei are stained with DAPI (blue), FISH EGFR probe (red) label tumor cells carrying EGFR amplification as double minutes (display multiple red signals). (b) Endothelial cells are detected by anti-CD31 immunofluorescence staining. ((c), (d)) A CD31+ (green) endothelial cell (arrow) carrying EGFR amplification (multiple red signals) is visible in a GBM microvessel.

VE-cadherin endothelial markers even after growth in EGM2 medium, known to promote endothelial differentiation [21] (Figure 2(c)).

To test the hypothesis that GSC might need a more specific microenvironment for endothelial conversion, we cocultured GSC-GFP with different human endothelial cells (HUVEC, HUAEC, and hCMEC) allowing cell-cell contact. In this condition, some GSC-GFP exhibited cobblestone morphology and expressed specific endothelial markers including CD31, CD105, VE-cadherin, and vWF (Figure 3(a)). The endothelial-like GSC-GFP were found consistently, but their percentage was variable $(0.2 \%$ to $1 \%)$ depending on the GSC and the endothelial cell types used in direct coculture (Figure 3(b)). However, this conversion was not observed in noncontact coculture, suggesting the implication of cell fusion or cell contact factors.

3.3. Some Glioblastoma Stem Cells Acquire the Endothelial Phenotype through Cell Fusion. The fact that most endothelial-like tumor cells were multinucleated suggested that GSC may acquire endothelial phenotype through cell fusion, rather than cell transdifferentiation. To test this hypothesis, we used DsRed protein-expressing HUVEC to detect cell fusion in fluorescent microscopy. When GSCGFP were cocultured with HUVEC-DsRed, binucleated cells coexpressing both GFP and DsRed were observed (Figure 4(a), (A)-(C)). GFP+/DsRed+ fused cells maintained CD31 expression (Figure 4(a), (D)), meaning that the endothelial phenotype was dominant. We isolated the GFP+/DsRed+ cells by cell sorting and cultured them in EGM-2 medium to monitor their behavior over time. Most of these cells with heterocaryons were quiescent and died after 5 to 7 days of culture. A small fraction (less than 1\%) gave rise to viable mononuclear hybrids expressing the parental GFP and DsRed, as shown in Figure 4(b).

\section{Discussion}

As early as 1948, several observations suggested that cancer cells are located in the walls of tumor blood vessels and participate in their assembly $[22,23]$. This is known as "mosaic vessels" where tumor cells form a part of the vessel surface, while the remaining part is covered by endothelium [24]. But in this case, tumor cells in apparent contact with the lumen do not show an endothelial phenotype. We report here that human GBM contain some tumor endothelial cells carrying the same cytogenetic abnormality as tumor cells. This phenomenon was reported previously in B-cell lymphomas [13], multiple myeloma [14], and neuroblastoma [15]. Since a common precursor can give rise to hematopoietic lineage and endothelial cells in hematopoietic tumors, a common progenitor targeted by neoplastic transformation and sharing specific genetic abnormalities can differentiate into tumor cells or endothelial cells [13, 14]. This hypothesis is not applicable to solid tumors of nonhematopoietic origin. Alternative explanations include tumor microenvironment 

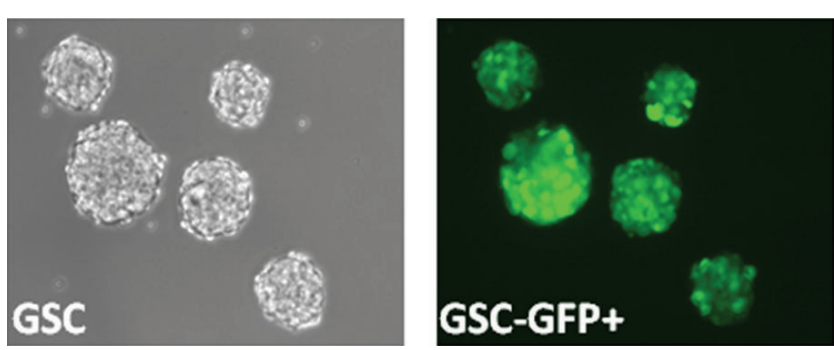

(a)

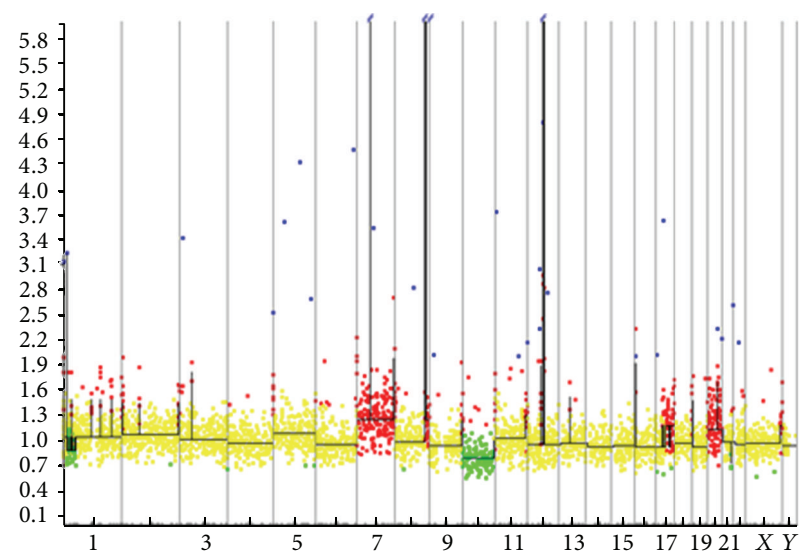

(b)

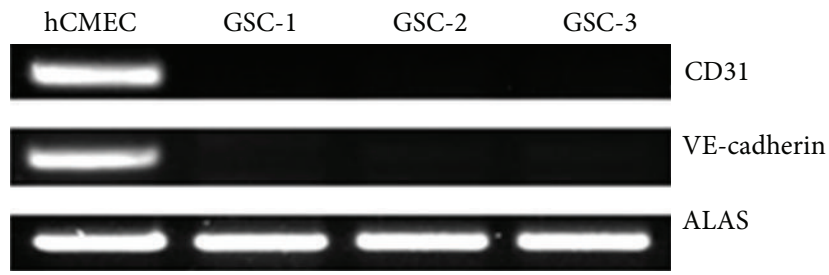

(c)
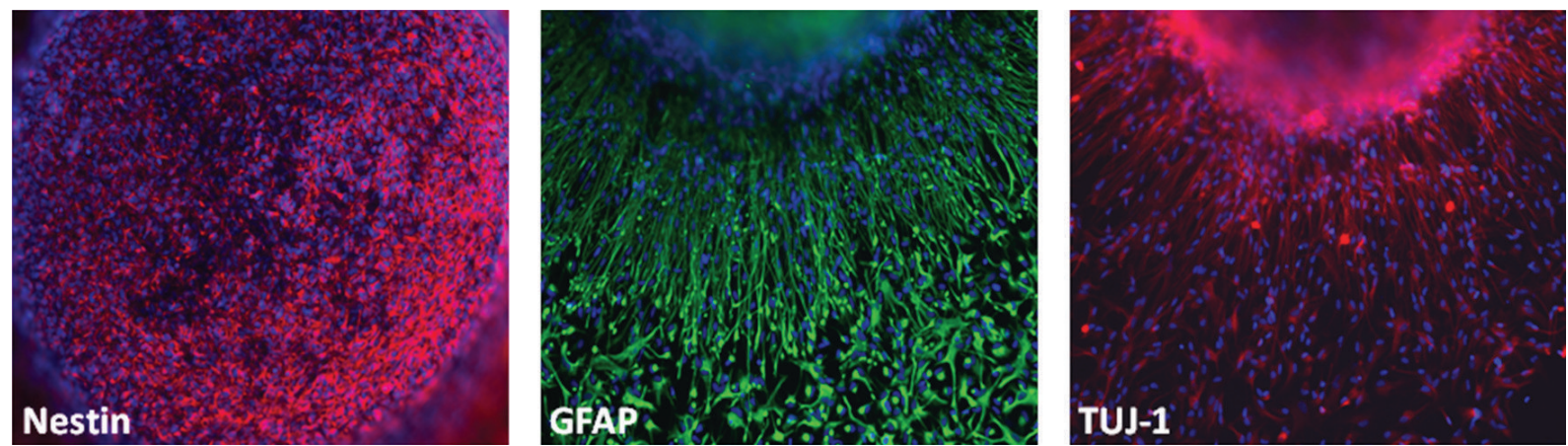

(d)

FIGURE 2: Glioblastoma stem cell characterization. (a) Phase contrast and fluorescent microscopy of GSC-1-GFP+ growing into tumor spheres in neurosphere medium (magnification $\times 10$ ). (b) Comparative genomic hybridization array (CGHa) of GSC-1 demonstrating tumor genomic alterations. Each BAC (bacterial artificial chromosome) spotted on the comparative genomic hybridization array is represented by a dot. BACs are ordered on the $x$-axis according to their position in the genome. For each chromosome, the telomere of the short arm is on the left and the telomere of the long arm is on the right. The $y$-axis corresponds to fluorescence ratio. Yellow, green, and red indicate, respectively, genomic copy number normal, loss, and gain. Genetic alteration includes complete chromosome 10 loss (green) and gain of chromosome 7 with EGFR amplification (arrow). (c) RT-PCR analysis showing specific expression of endothelial markers CD31 and VE-cadherin by hCMEC and not by GSC-1, GSC-2, and GSC-3 after differentiation in EGM-2 medium. ALAS is used as control. (d) Immunostaining of tumor sphere cells for neural stem cell marker (nestin) at the beginning of the differentiation assay, then astrocytic (GFAP) and neuronal (TUJ-1) markers by the differentiated cells around a tumor sphere at day 7 (magnification $\times 10)$.

[25, 26], tumor cell transdifferentiation, and cell fusion [27]. Our data strongly suggest that GBM stem cell acquires endothelial phenotype through cell fusion and participates in microvascular structures.

This is consistent with a recent study in which human glioma lines marked with DsRed protein were grafted in eGFP-expressing NOD/Scid mouse to study tumor-host interactions [28]. Using fluorescence-activated cell sorting (FACS), this model is allowed to completely separate host cells from tumor cells and to detect double-positive cells, possibly arising from cell fusion events. Furthermore, CD31 expression was found in $0.3 \%$ of separated tumor cells when phenotyped [28]. In normal brain, neural stem cells exist in vascular niches where endothelial cells provide direct cell contacts and secreted factors that regulate neural stem cell function $[29,30]$. Similarly, GBM stem cells are maintained within vascular niches that mimic the neural stem cell niche [31]. Nestin+/CD133+ cells within sections of human GBM 


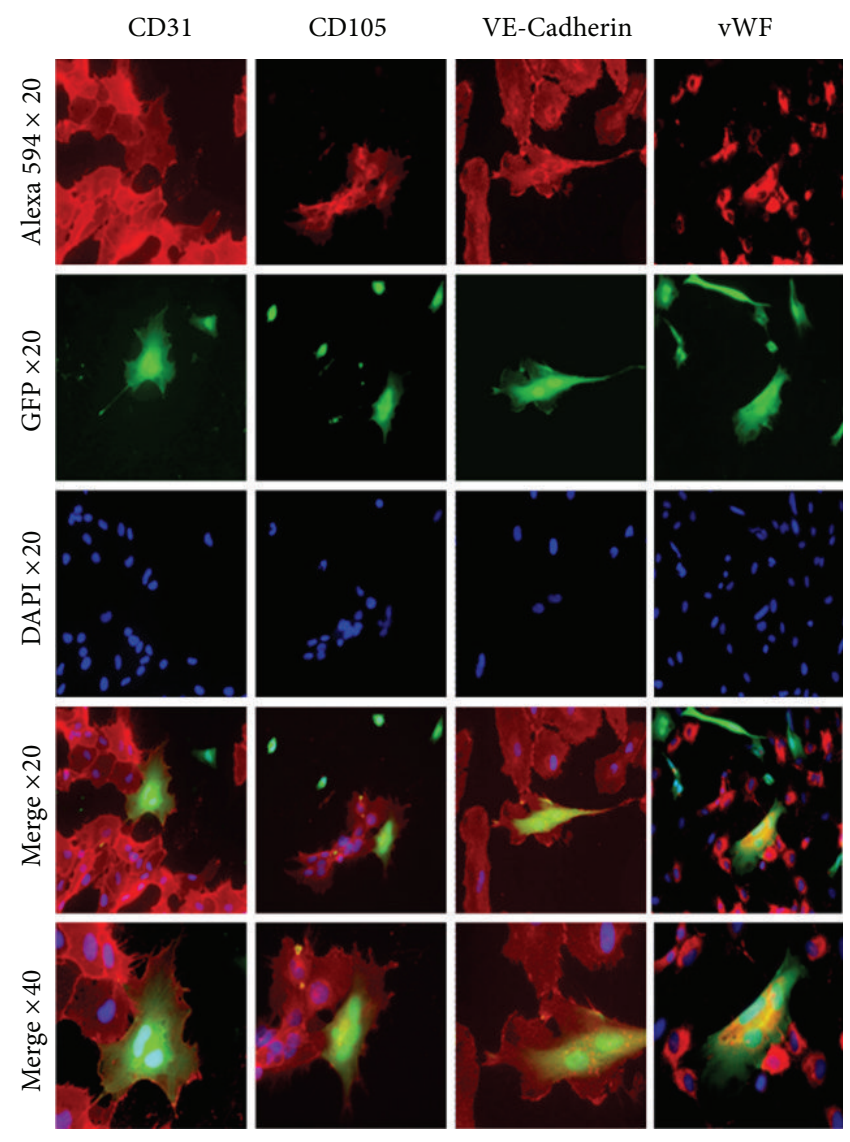

(a)

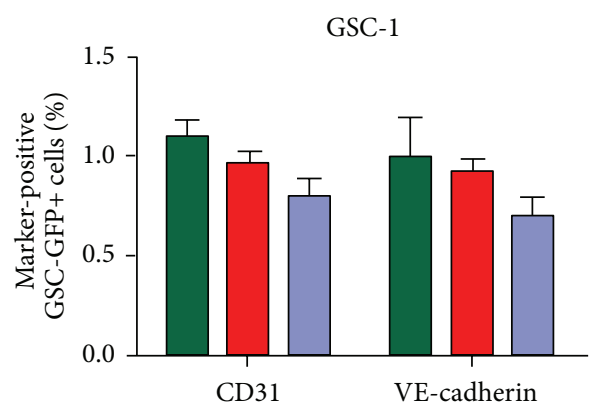

GSC-2
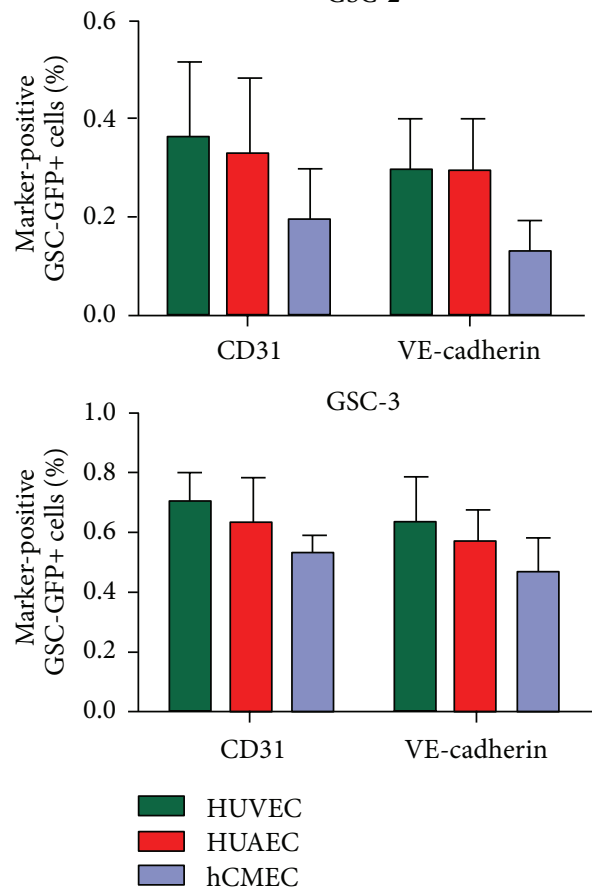

GSC-3

(b)

FIGURE 3: A subset of glioblastoma stem cells acquires an endothelial phenotype. (a) When GSC-GFP were cocultured with human endothelial cells, several multinucleated and cobblestone GSC-GFP expressing CD31, CD105, VE-cadherin, and vWF were observed (b). The percentage of GSC-GFP expressing CD31 and VE-cadherin obtained in coculture with HUVEC, HUAEC, and hCMEC is indicated for GSC-1, GSC-2, and GSC-3.

are located in close proximity to tumor capillaries [31]. This organization can favor cell fusion by facilitating cell contact between GBM stem cells and endothelial cells.

Cell fusion has been debated in adult stem cells plasticity. Several reports revealed the ability for stem cells to change fate through cell fusion rather than cell transdifferentiation. New hybrids resulting from nuclear fusion can generate mononuclear differentiated progeny that exhibit both parental phenotypes [18, 32-35]. These findings raise the possibility that cell fusion has undiscovered functions which can promote disease, especially cancer. The idea that cell fusion contributes to cancer progression was introduced almost 100 years ago with a proposal that malignancy is a consequence of hybridization between leukocytes and somatic cells [36]. Sixty years later, this idea was expanded by proposing that hybridization of tumor cells with lymphocytes results in metastatic cells [37] and that cell fusion promotes the phenotypic and genotypic diversity of tumors
[38]. The best defended theory is cancer cell fusion with macrophages or other migratory bone marrow-derived cells which provides a unifying explanation for metastasis [39]. Although host cell-cancer cell fusion has been demonstrated and well documented in animals [40], there is as yet far less information on human cancer. A few human cases have recently been reported [41-45], but it is unclear whether the scarcity of reports on cell fusion in human tumors is due to rarity of this event or to a difficulty to detect it.

In addition to cell fusion, we cannot exclude that transdifferentiation of GBM stem cells into endothelial cells may exist. Conversion from GBM to endothelial phenotype was not observed in noncontact coculture, suggesting either cell fusion or cell-cell contact mediated transdifferentiation. Recently two recent papers showed that transdifferentiation of GBM stem cells into endothelial cells does exist in GBM (p7). Notch pathway is involved in this mechanism [46-48]. 


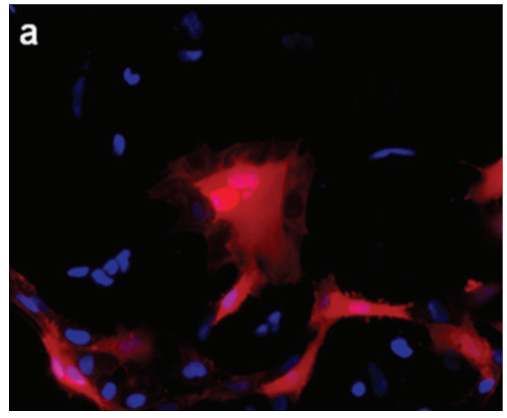

(A)

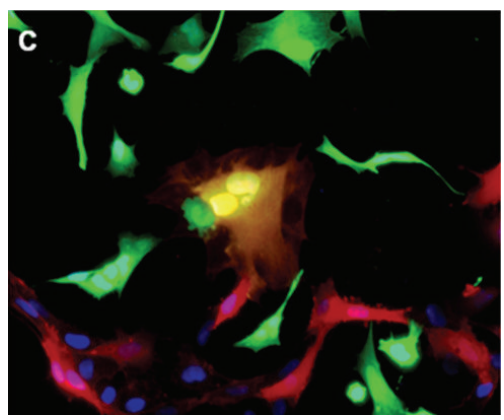

(C)

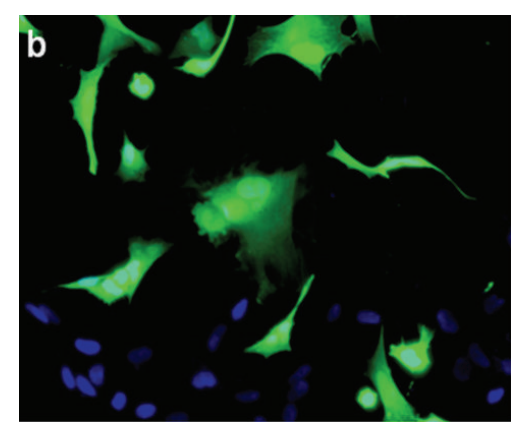

(B)

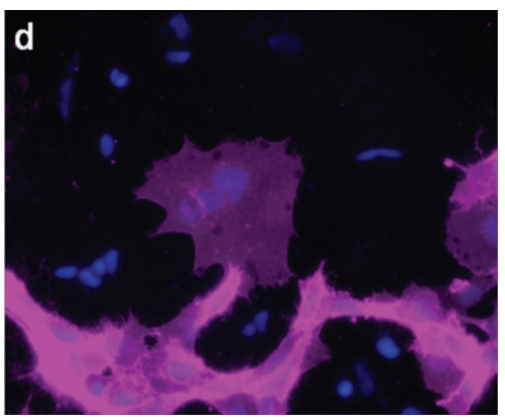

(D)

(a)

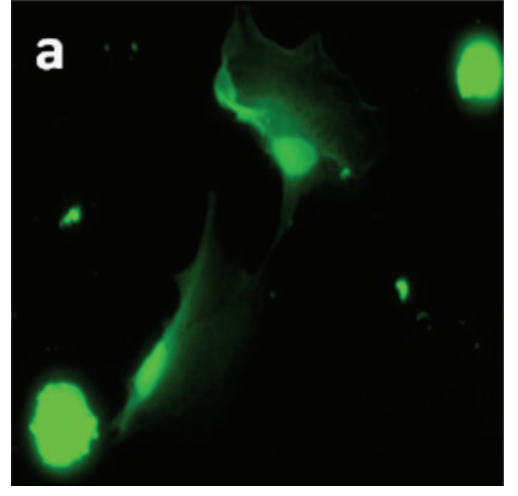

(A)

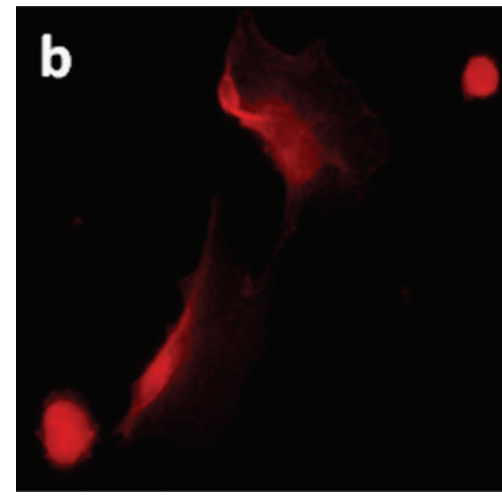

(B)

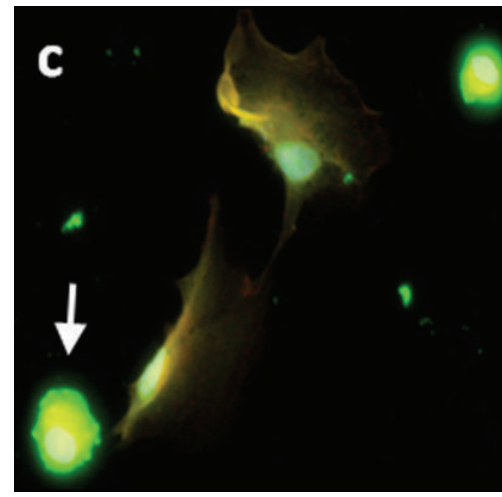

(C)

(b)

FIGURE 4: Glioblastoma stem cell fusion with endothelial cells. (a) When GSC-GFP were cocultured with HUVEC-DsRed, we observed binucleated cell expressing both DsRed (A) and GFP (B) (merged in (C)) corresponding to hybrid cell and exhibiting endothelial phenotype as shown by CD31 immunostaining (D). (b) After hybrid cell selection by cell sorting, mononuclear hybrids expressing parental GFP (A) and DsRed (B) were observed (merged in (C)). Apoptotic body of died fused cell (arrow).

Considering that cell fusion can be linked to several fundamental features of cancer, more investigations are needed to clarify this emergent concept of "hidden enemy" in cancer pathology. In addition, the presence of these hybrid cells in GBM endothelium supports new therapeutic approaches, such as intravascular targeted strategy directed against the EGFRVIII mutant, a specific antigen expressed by $20-30 \%$ of GBM [49].

\section{Conflict of Interests}

The authors declare that there is no conflict of interests regarding the publication of this paper.

\section{Acknowledgments}

This work is supported by a Grant from the Institut National du Cancer (PL 046) and the Institut de France (Fondation 
Energie). S. El Hallani and C. Colin were supported by the Ligue Nationale Contre le Cancer.

\section{References}

[1] D. N. Louis, H. Ohgaki, O. D. Wiestler et al., "The 2007 WHO classification of tumours of the central nervous system," Acta Neuropathologica, vol. 114, no. 2, pp. 97-109, 2007.

[2] J. J. Vredenburgh, A. Desjardins, J. E. Herndon II et al., "Phase II trial of bevacizumab and irinotecan in recurrent malignant glioma," Clinical Cancer Research, vol. 13, no. 4, pp. 1253-1259, 2007.

[3] T. N. Kreisl, L. Kim, K. Moore et al., "Phase II trial of singleagent bevacizumab followed by bevacizumab plus irinotecan at tumor progression in recurrent glioblastoma," Journal of Clinical Oncology, vol. 27, no. 5, pp. 740-745, 2009.

[4] D. M. McDonald and P. L. Choyke, "Imaging of angiogenesis: from microscope to clinic," Nature Medicine, vol. 9, no. 6, pp. 713-725, 2003.

[5] F. Mollica, R. K. Jain, and P. A. Netti, "A model for temporal heterogeneities of tumor blood flow," Microvascular Research, vol. 65, no. 1, pp. 56-60, 2003.

[6] D. M. McDonald and P. Baluk, "Significance of blood vessel leakiness in cancer," Cancer Research, vol. 62, no. 18, pp. 53815385, 2002.

[7] P. Baluk, S. Morikawa, A. Haskell, M. Mancuso, and D. M. McDonald, "Abnormalities of basement membrane on blood vessels and endothelial sprouts in tumors," The American Journal of Pathology, vol. 163, no. 5, pp. 1801-1815, 2003.

[8] B. St. Croix, C. Rago, V. Velculescu et al., "Genes expressed in human tumor endothelium," Science, vol. 289, no. 5482, pp. 1197-1202, 2000.

[9] B. Bussolati, I. Deambrosis, S. Russo, M. C. Deregibus, and G. Camussi, "Altered angiogenesis and survival in human tumorderived endothelial cells," The FASEB Journal, vol. 17, no. 9, pp. 1159-1161, 2003.

[10] K. Hida and M. Klagsbrun, "A new perspective on tumor endothelial cells: unexpected chromosome and centrosome abnormalities," Cancer Research, vol. 65, no. 7, pp. 2507-2510, 2005.

[11] C. Grange, B. Bussolati, S. Bruno, V. Fonsato, A. Sapino, and G. Camussi, "Isolation and characterization of human breast tumor-derived endothelial cells," Oncology Reports, vol. 15, no. 2, pp. 381-386, 2006.

[12] K. Hida, Y. Hida, D. N. Amin et al., "Tumor-associated endothelial cells with cytogenetic abnormalities," Cancer Research, vol. 64, no. 22, pp. 8249-8255, 2004.

[13] B. Streubel, A. Chott, D. Huber et al., "Lymphoma-specific genetic aberrations in microvascular endothelial cells in B-cell lymphomas," The New England Journal of Medicine, vol. 351, no. 3, pp. 250-259, 2004.

[14] G. M. Rigolin, C. Fraulini, M. Ciccone et al., "Neoplastic circulating endothelial cells in multiple myeloma with 13q14 deletion," Blood, vol. 107, no. 6, pp. 2531-2535, 2006.

[15] A. Pezzolo, F. Parodi, M. V. Corrias, R. Cinti, C. Gambini, and V. Pistoia, "Tumor origin of endothelial cells in human neuroblastoma," Journal of Clinical Oncology, vol. 25, no. 4, pp. 376-383, 2007.

[16] D. Bonnet and J. E. Dick, "Human acute myeloid leukemia is organized as a hierarchy that originates from a primitive hematopoietic cell," Nature Medicine, vol. 3, no. 7, pp. 730-737, 1997.
[17] S. K. Singh, I. D. Clarke, M. Terasaki et al., "Identification of a cancer stem cell in human brain tumors," Cancer Research, vol. 63, no. 18, pp. 5821-5828, 2003.

[18] N. Terada, T. Hamazaki, M. Oka et al., "Bone marrow cells adopt the phenotype of other cells by spontaneous cell fusion," Nature, vol. 416, no. 6880, pp. 542-545, 2002.

[19] A. Idbaih, Y. Marie, C. Lucchesi et al., "BAC array CGH distinguishes mutually exclusive alterations that define clinicogenetic subtypes of gliomas," International Journal of Cancer, vol. 122, no. 8, pp. 1778-1786, 2008.

[20] H. A. Russ, Y. Bar, P. Ravassard, and S. Efrat, "In vitro proliferation of cells derived from adult human $\beta$-cells revealed by cell-lineage tracing," Diabetes, vol. 57, no. 6, pp. 1575-1583, 2008.

[21] S. Heydarkhan-Hagvall, K. Schenke-Layland, J. Q. Yang et al., "Human adipose stem cells: a potential cell source for cardiovascular tissue engineering," Cells Tissues Organs, vol. 187, no. 4, pp. 263-274, 2008.

[22] R. A. Willis, Pathology of Tumour, Butterworth Heinemann, Oxford, UK, 1948.

[23] F. Hammersen, B. Endrich, and K. Messmer, "The fine structure of tumor blood vessels. I. Participation of non-endothelial cells in tumor angiogenesis," International Journal of Microcirculation, Clinical and Experimental, vol. 4, no. 1, pp. 31-43, 1985.

[24] Y. S. Chang, E. di Tomaso, D. M. McDonald, R. Jones, R. K. Jain, and L. L. Munn, "Mosaic blood vessels in tumors: frequency of cancer cells in contact with flowing blood," Proceedings of the National Academy of Sciences of the United States of America, vol. 97, no. 26, pp. 14608-14613, 2000.

[25] B. Vogelstein and K. W. Kinzler, "Cancer genes and the pathways they control," Nature Medicine, vol. 10, no. 8, pp. 789-799, 2004.

[26] N. Ferrara and R. S. Kerbel, "Angiogenesis as a therapeutic target," Nature, vol. 438, no. 7070, pp. 967-974, 2005.

[27] K. Mortensen, J. Lichtenberg, P. D. Thomsen, and L.-I. Larsson, "Spontaneous fusion between cancer cells and endothelial cells," Cellular and Molecular Life Sciences, vol. 61, no. 16, pp. 2125-2131, 2004.

[28] S. P. Niclou, C. Danzeisen, H. P. Eikesdal et al., "A novel eGFPexpressing immunodeficient mouse model to study tumor-host interactions," The FASEB Journal, vol. 22, no. 9, pp. 3120-3128, 2008.

[29] T. D. Palmer, A. R. Willhoite, and F. H. Gage, "Vascular niche for adult hippocampal neurogenesis," Journal of Comparative Neurology, vol. 425, no. 4, pp. 479-494, 2000.

[30] Q. Shen, S. K. Goderie, L. Jin et al., "Endothelial cells stimulate self-renewal and expand neurogenesis of neural stem cells," Science, vol. 304, no. 5675, pp. 1338-1340, 2004.

[31] C. Calabrese, H. Poppleton, M. Kocak et al., "A perivascular niche for brain tumor stem cells," Cancer Cell, vol. 11, no. 1, pp. 69-82, 2007.

[32] Q.-L. Ying, J. Nichols, E. P. Evans, and A. G. Smith, "Changing potency by spontaneous fusion," Nature, vol. 416, no. 6880, pp. 545-548, 2002.

[33] J. L. Spees, S. D. Olson, J. Ylostalo et al., "Differentiation, cell fusion, and nuclear fusion during ex vivo repair of epithelium by human adult stem cells from bone marrow stroma," Proceedings of the National Academy of Sciences of the United States of America, vol. 100, no. 5, pp. 2397-2402, 2003.

[34] G. Vassilopoulos, P.-R. Wang, and D. W. Russell, "Transplanted bone marrow regenerates liver by cell fusion," Nature, vol. 422, no. 6934, pp. 901-904, 2003. 
[35] X. Wang, H. Willenbring, Y. Akkari et al., "Cell fusion is the principal source of bone-marrow-derived hepatocytes," Nature, vol. 422, no. 6934, pp. 897-901, 2003.

[36] O. Aichel, Vorträge und Aufsätze über Entvickelungsmechanik der Organismen, chapter XIII, Wilhelm Engelmann, Leipzig, Germany, 1911.

[37] L. B. Mekler, "Hybridization of transformed cells with lymphocytes as 1 of the probable causes of the progression leading to the development of metastatic malignant cells," Vestnik Akademii Meditsinskikh Nauk SSSR, vol. 26, no. 8, pp. 80-89, 1971.

[38] T. F. C. S. Warner, "Cell hybridization: an explanation for the phenotypic diversity of certain tumours," Medical Hypotheses, vol. 1, no. 1, pp. 51-57, 1975.

[39] J. M. Pawelek and A. K. Chakraborty, "Fusion of tumour cells with bone marrow-derived cells: a unifying explanation for metastasis," Nature Reviews Cancer, vol. 8, no. 5, pp. 377-386, 2008.

[40] D. Duelli and Y. Lazebnik, "Cell fusion: a hidden enemy?" Cancer Cell, vol. 3, no. 5, pp. 445-448, 2003.

[41] T. L. Andersen, P. Boissy, T. E. Sondergaard et al., "Osteoclast nuclei of myeloma patients show chromosome translocations specific for the myeloma cell clone: a new type of cancer-host partnership?" The Journal of Pathology, vol. 211, no. 1, pp. 10-17, 2007.

[42] A. Chakraborty, R. Lazova, S. Davies et al., "Donor DNA in a renal cell carcinoma metastasis from a bone marrow transplant recipient," Bone Marrow Transplantation, vol. 34, no. 2, pp. 183186, 2004.

[43] Y. Yilmaz, R. Lazova, M. Qumsiyeh, D. Cooper, and J. Pawelek, "Donor Y chromosome in renal carcinoma cells of a female BMT recipient: visualization of putative BMT-tumor hybrids by FISH," Bone Marrow Transplantation, vol. 35, no. 10, pp. 10211024, 2005.

[44] C. R. Cogle, N. D. Theise, D. Fu et al., "Bone marrow contributes to epithelial cancers in mice and humans as developmental mimicry," Stem Cells, vol. 25, no. 8, pp. 1881-1887, 2007.

[45] I. Avital, A. L. Moreira, D. S. Klimstra et al., "Donor-derived human bone marrow cells contribute to solid organ cancers developing after bone marrow transplantation," Stem Cells, vol. 25, no. 11, pp. 2903-2909, 2007.

[46] L. Ricci-Vitiani, R. Pallini, M. Biffoni et al., "Tumour vascularization via endothelial differentiation of glioblastoma stem-like cells," Nature, vol. 468, no. 7325, pp. 824-828, 2010.

[47] R. Wang, K. Chadalavada, J. Wilshire et al., "Glioblastoma stemlike cells give rise to tumour endothelium," Nature, vol. 468, no. 7325, pp. 829-835, 2010.

[48] Y. Soda, T. Marumoto, D. Friedmann-Morvinski et al., "Transdifferentiation of glioblastoma cells into vascular endothelial cells," Proceedings of the National Academy of Sciences of the United States of America, vol. 108, no. 11, pp. 4274-4280, 2011.

[49] H. K. Gan, A. H. Kaye, and R. B. Luwor, "The EGFRvIII variant in glioblastoma multiforme," Journal of Clinical Neuroscience, vol. 16, no. 6, pp. 748-754, 2009. 

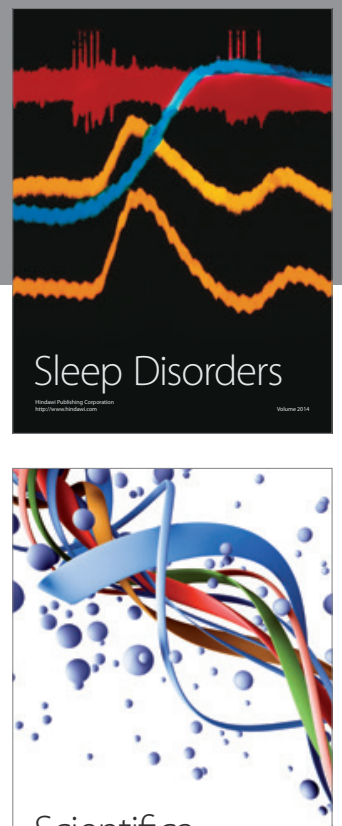

Scientifica
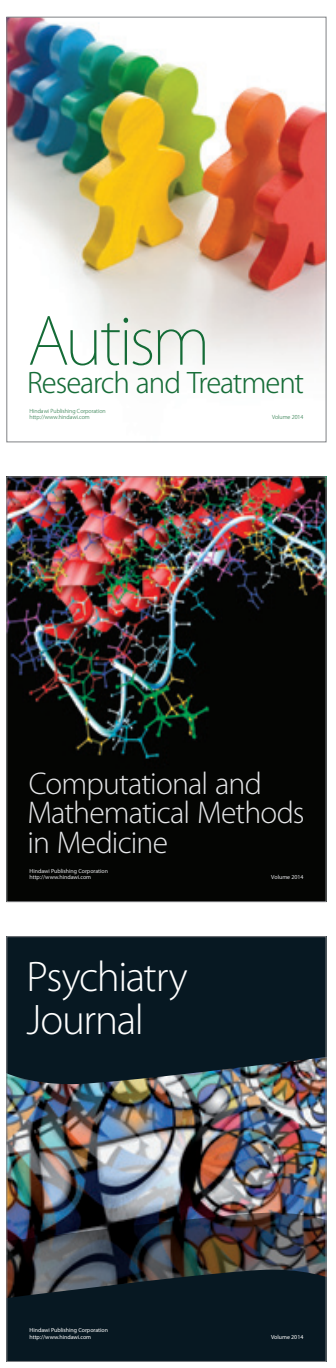
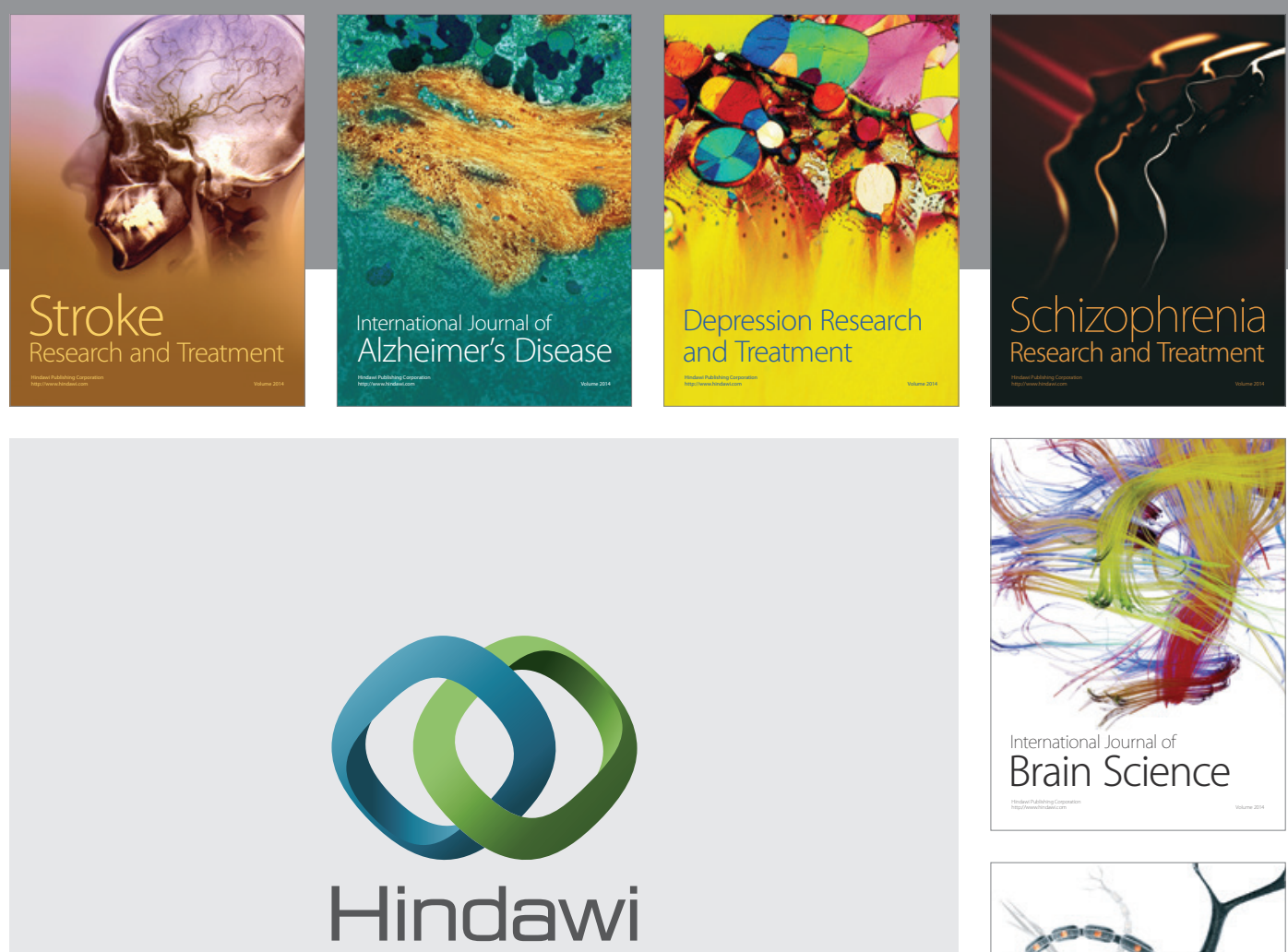

Submit your manuscripts at

http://www.hindawi.com
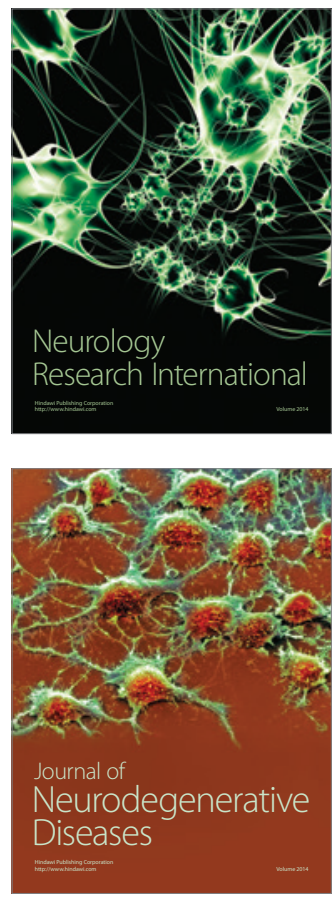

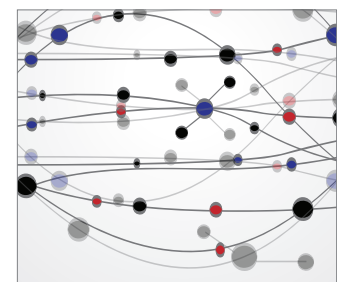

The Scientific World Journal
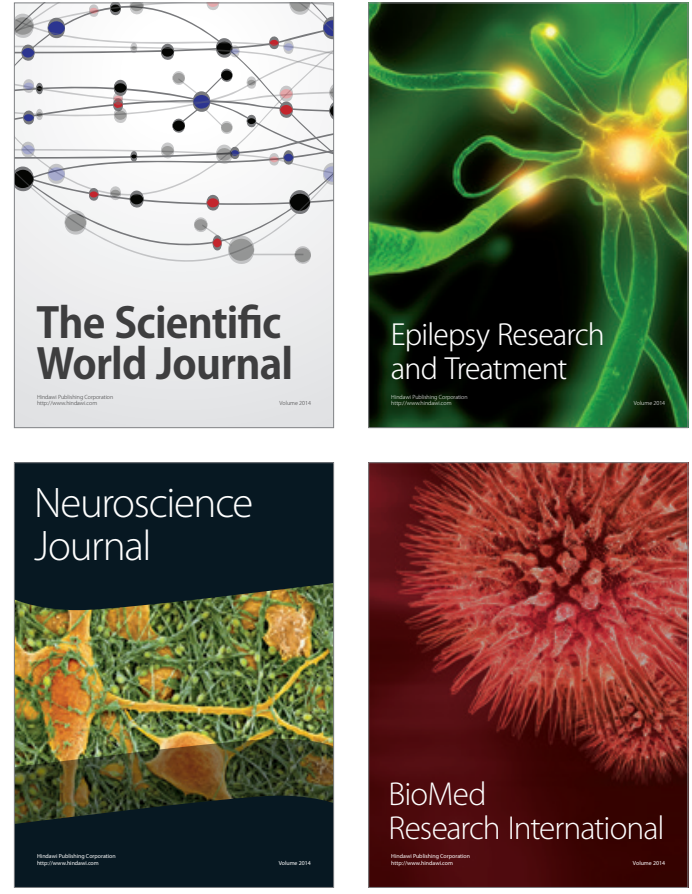

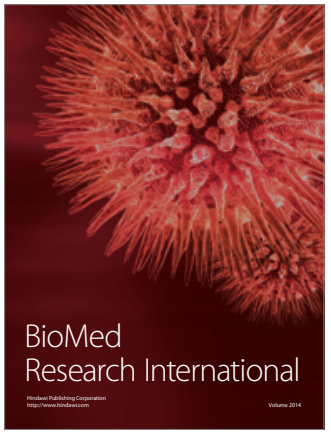

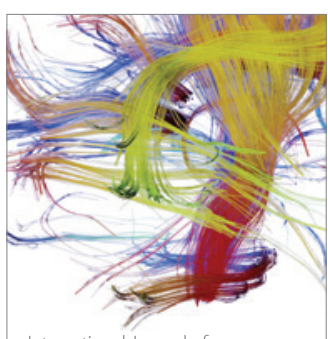

Brain Science

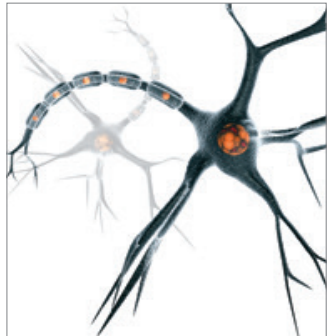

Neural Plasticity
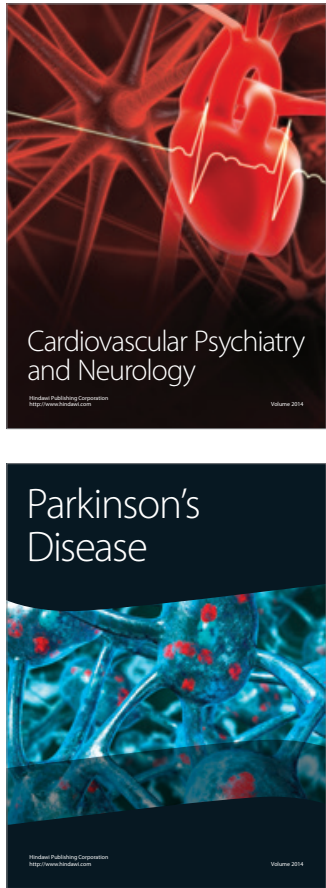